\title{
The effect of diagnostic hysteroscopy performed before fresh and frozen-thawed embryo transfer in IVF cycles on reproductive outcomes
}

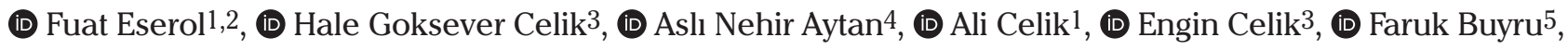 \\ (D) Ercan Bastu 5 \\ ${ }^{1}$ Clinic of Obstetrics and Gynecology, Batı Bahat Hospital, İstanbul, Turkey \\ 2Department of Obstetrics and Gynecology, İstanbul Yeni Yüzyll University Faculty of Medicine, İstanbul, Turkey \\ ${ }^{3}$ Clinic of Obstetrics and Gynecology, University of Health Sciences Turkey, Kanuni Sultan Süleyman Training and Research \\ Hospital, İstanbul, Turkey \\ 4Department of Obstetrics and Gynecology, Bahçeşehir University Faculty of Medicine, İstanbul, Turkey \\ 5Department of Obstetrics and Gynecology, Acıbadem Mehmet Ali Aydınlar University Faculty of Medicine, İstanbul, Turkey
}

\section{Abstract}

Objective: Hysteroscopy is frequently performed in infertile women and thought to improve pregnancy rates. The data obtained from studies investigating the effect of hysteroscopy in in-vitro fertilization (IVF) cycles is variable. We aimed to evaluate the effect of hysteroscopy on pregnancy outcomes of fresh and frozen-thawed embryo transfers (FET) performed during IVF cycles.

Material and Methods: The data of the 765 patients, who had IVF treatment between January 2015 and July 2017 in an infertility center, were retrospectively analyzed. Of those, 586 (76.6\%) patients underwent fresh embryo transfer, while 179 (23.4\%) patients underwent FET. Hysteroscopy performed by a single experienced surgeon was scheduled two months before transfer. Hysteroscopy was performed in $101 / 586$ (17.2\%) in those undergoing fresh embryo transfer and 44/179 (24.6\%) patients in the FET group. Pregnancy outcomes of the groups were compared respectively within their own group.

Results: The mean age was similar in patients in the fresh and FET groups ( $p=0.365$, respectively). There was no difference in the number of transferred embryos between the groups $(p=0.218)$. In the fresh embryo group there were 246 pregnancies, of which 44 had undergone diagnostic hysteroscopy while 202 had not, $(\mathrm{p}=0.516)$ and 79 pregnancies in the FET group, of which 20 had undergone diagnostic hysteroscopy while 59 had not $(\mathrm{p}=0.711)$. There was no statistical difference according to pregnancy rate between the groups $(\mathrm{p}=0.538)$.

Conclusion: Performing diagnostic hysteroscopy before fresh or FET does not improve the pregnancy rates.(J Turk Ger Gynecol Assoc 2021; 22: 206-11)

Keywords: Diagnostic hysteroscopy, in-vitro fertilization, frozen-thawed embryo transfer, fresh embryo transfer, uterine cavity, pregnancy rate

Received: 03 August, 2020 Accepted: 05 January, 2021

\section{Introduction}

In-vitro fertilization (IVF) has given hope to infertile couples in the $20^{\text {th }}$ century. However, live birth occurs in only one third of IVF and intracytoplasmic sperm injection (ICSI) cycles (1). This low success rate is thought to be due to the failure of implantation. The exact reason underlying this implantation failure is not understood, and may depend on uterine cavity factors, embryo quality or a combination of these $(2,3)$. An abnormal uterine finding such as polyps, uterine leiomyoma, and adhesions are present in approximately 50\% of infertile women (4,5). Abnormalities of the uterine cavity may lead to implantation failure, in turn resulting in reduced chance of successful pregnancy outcome. Hysterosalpingogram (HSG), 
saline infusion sonohysterography (SIS) or hysteroscopy are alternative methods to evaluate the uterine cavity. The false positive rate and the false negative rate for HSG are 15.6\% and $35.4 \%$, respectively. Additionally; HSG offers no chance of management for the abnormalities of the uterine cavity. To obtain enhanced endometrial visualization, saline fluid is introduced into the uterine cavity transcervically during transvaginal ultrasound examination. Although this method is feasible and highly sensitive and specific when used for detection of endometrial abnormalities (97.3\% and 95.8\%, respectively), SIS, like HSG, does not provide a possibility for management of the abnormalities of the uterine cavity (6). Hysteroscopy is a more powerful technique to evaluate the uterine cavity and apply treatment simultaneously $(7,8)$. This makes hysteroscopy the most useful test for assessing the uterine cavity. The evaluation of uterine cavity with hysteroscopy is especially valuable in women with prior IVF failures (9).

Studies evaluating the usefulness of hysteroscopy in IVF cycles have produced conflicting results. The TROPHY study, a multicenter, randomized controlled trial reported that, especially for women with recurrent, unsuccessful implantation following IVF, hysteroscopy had no effect on the live birth rate (LBR) (10). However, this contrasts with the results of a prior systematic review suggesting that routine hysteroscopy improved the LBR for women with recurrent unsuccessful IVF cycles (11). Comparing the performance of hysteroscopy with no hysteroscopy prior to any (first or subsequent) IVF/ICSI attempt in infertile aymptomatic patients, there was very limited evidence suggesting that hysteroscopy was useful to increase LBR (12). Similar to the results of the TROPHY study, another multicentre, randomised controlled trial published in the same year, the INSIGHT trial, reported that LBR in infertile women with a normal uterine cavity on transvaginal ultrasound has not been improved by applying routine diagnostic hysteroscopy prior to the first IVF treatment (13). Pabuçcu et al. (14) also found that hysteroscopy did not make a statistically significant difference regarding implantation, pregnancy rate and LBR in infertile women having a history of recurrent implantation failure.

Another important issue that has been investigated is that of endometrial scratching during hysteroscopy before IVF treatment. Endometrial scratching results in superficial injury of uterine cavity, which is thought to enhance the receptivity of the uterus for the embryo (15). Subsequent studies have reported that endometrial damage did not increase pregnancy rates (16).

In this study, pregnancy outcomes of IVF cycles were evaluated when the embryo transfer was either fresh or frozen-thawed embryo that were applied with or without hysteroscopy to evaluate the pre-IVF uterine cavity.

\section{Material and Methods}

\section{Participants}

The data of 768 patients who underwent IVF cycles between January 2015 and July 2017 in a private infertility center was evaluated retrospectively. For this study, ethical approval was taken from the Ethical Committee of Acıbadem Mehmet Ali Aydınlar University. All patients who participated in this study gave written informed consent for this study. Women aged 1845 years with primary infertility due to tubal factor, male factor or unexplained were selected for the study. Patients who had infertility with known uterine factors and recurrent miscarriage were excluded from the study. Transvaginal ultrasound of the endometrial cavity, HSG, and SIS were performed and patients having any pathology were also excluded from the study. Only women with no pathology detected by hysteroscopy were included in the study.

Fresh embryo transfer was used in 589 of these patients while frozen-thawed embryo transfer (FET) was performed in 179 patients. Each of these two groups was separated into two new groups, depending on if the subjects had undergone diagnostic hysteroscopy or not. Hysteroscopy was performed two months prior to IVF in women with suspected structural lesions in the uterine cavity before the embryo transfer by a single experienced surgeon. A total of 101 (17.2\%) of the patients in the group of fresh embryo transfer and $44(24.6 \%)$ in the FET group underwent hysteroscopy.

\section{Procedure}

Hysteroscopy was performed during the early proliferative phase in the outpatient clinic without anesthesia using by a 1.9 $\mathrm{mm}$ Karl Storz hysteroscope with a $30^{\circ}$ view. Saline distension medium was used. A paracervical block was applied in the patients with intolerance. Patients were hospitalized only for 15-60 min and no complications were experienced.

Controlled ovarian stimulation $(\mathrm{COH})$ was achieved using human menopausal gonadotropin (Merional 75 IU, IBSA Institut, Switzerland) with the adjusted dose based on the individual response and human chorionic gonadotropin ( $\mathrm{hCG}$ ) at a dose of 10,000 IU in the fresh embryo transfer group. $\mathrm{COH}$ was begun at the time of menses as antagonist protocol. An antagonist was administered when the follicles became greater than $14 \mathrm{~mm}$ in largest diameter and daily injections of the antagonist were continued until hCG administration. After oocyte retrieval and fertilization, embryo transfers were performed on day three. Frozen-thawed embryo protocol was used in the other group. For patients with thin endometrium, hormone treatment was offered to prepare the endometrium ( $2 \mathrm{mg}$ micronized estradiol tablet). After the endometrial thickness in each patient became greater than $8 \mathrm{~mm}$, progesterone therapy (Progestan $200 \mathrm{mg}$; 
Koçak, Tekirdağ, Turkey) three times a day via vaginal pathway or daily progesterone gel (Crinone $8 \%$, Merck Serono, Italy) was added to treatment at the $14^{\text {th }}$ day of cycles. Upon completion of endometrial preparation, the transfer of a day 3 embryo (cleavage stage) was performed on the third day. Although the number of embryos transferred changed depending on a number of factors, such as maternal age, the number of oocytes retrieved and availability of embryos for cryopreservation, no more than two embryos was transferred in our population. While selecting the subjects for different groups, an attempt was made to keep the number of transferred embryos alike.

Beta hCG values were determined from blood samples of the patients after 11 days from embryo transfer and results over 10 $\mathrm{mIU} / \mathrm{mL}$ were accepted as pregnancy.

\section{Statistical analysis}

Statistical analysis was performed with the Statistical Package for the Social Sciences (IBM Inc., Chicago, IL, USA) version 16.0. Difference in mean values and characteristics between groups was analyzed with Independent samples t-test and chisquare test. Means were presented with standard deviation. A $\mathrm{p}<0.05$ was accepted as statistically significant.

\section{Results}

The total cohort numbered 765 women, of whom fresh embryo transfer was done in 586 (76.6\%) while FET was performed in $179(23.4 \%)$. In the fresh embryo transfer group, diagnostic hysteroscopy was performed in 101 (17.2\%) and in the FET group this number was $44(24.6 \%)$. No pathological findings were found in any of the subjects during the hysteroscopic procedure, so no treatment was required. The clinicodemographic characteristics of the participants are presented in Table 1. The mean age of the patients was comparable between the patients in fresh and FET groups $(\mathrm{p}=0.365)$. There was no statistical difference in transferred embryo numbers in fresh and FET groups $(\mathrm{p}=0.218)$. The quality of the embryos was statistically similar in both groups $(\mathrm{p}=0.177)$.

In the fresh embryo group there were 246 pregnancies, of which 44 had undergone diagnostic hysteroscopy while 202 had not, $(\mathrm{p}=0.516)$ and 79 pregnancies in the FET group, of which 20 had undergone diagnostic hysteroscopy while 59 had not $(\mathrm{p}=0.711)$. There was no statistical difference in take-home baby rates between the groups in which hysteroscopy was performed and was not performed ( $\mathrm{p}=0.513)$ (Table 2). Table 3 presents the comparison of the patients, grouped by age. There was a significant difference between the patients regarding take-home baby rates, pregnancy results and obstetric outcomes ( $\mathrm{p}<0.001, \mathrm{p}=0.001$ and $\mathrm{p}=0.001$, respectively) when age was taken into account.

\section{Discussion}

Considering that the success rate of IVF and ICSI treatments is $25-30 \%$ and the most common reason for low success rate is implantation failure, the evaluation of the endometrial cavity prior to IVF procedures is undoubtedly very important (3). Hysteroscopy has been accepted as the "gold standard" test to evaluate the uterine cavity. Hysteroscopy also provides an opportunity for simultaneous treatment of any pathology detected during the procedure. Intrauterine lesions such as polyps, submucous myomas, and adhesions may be a significant factor resulting in implantation failure. Transvaginal ultrasound, SIS, and HSG may be insufficient to see small

\section{Table 1. Demographic and clinical characteristics of} the patients

\begin{tabular}{|c|c|}
\hline Characteristics & $\begin{array}{l}\text { Mean } \pm \text { SD or } \\
\text { number }(\%)\end{array}$ \\
\hline Age (years) & $31.2 \pm 6.0$ \\
\hline \multicolumn{2}{|l|}{ Age group (years) } \\
\hline$<20$ & $20(2.6)$ \\
\hline $21-25$ & $112(14.6)$ \\
\hline $26-30$ & $249(32.4)$ \\
\hline $31-35$ & $192(25)$ \\
\hline $36-40$ & $139(18.1)$ \\
\hline$>40$ & $56(7.3)$ \\
\hline \multicolumn{2}{|l|}{ Hysteroscopy } \\
\hline Not performed & $623(81.1)$ \\
\hline Performed & $145(18.9)$ \\
\hline \multicolumn{2}{|c|}{ Type of embryo transfer } \\
\hline Fresh & $589(76.7)$ \\
\hline Frozen-thawed & $179(23.3)$ \\
\hline \multicolumn{2}{|c|}{ Hysteroscopy regarding type of embryo transfer } \\
\hline \multicolumn{2}{|l|}{ Fresh embryo transfer } \\
\hline Not performed & $488(63.5)$ \\
\hline Performed & $101(13.2)$ \\
\hline \multicolumn{2}{|c|}{ Frozen-thawed embryo transfer } \\
\hline Not performed & $135(17.6)$ \\
\hline Performed & $44(5.7)$ \\
\hline \multicolumn{2}{|l|}{ Pregnancy result } \\
\hline Negative & $444(57.8)$ \\
\hline Positive & $324(42.2)$ \\
\hline \multicolumn{2}{|l|}{ Pregnancy outcome } \\
\hline Biochemical & $51(15.7)$ \\
\hline Abortus & $48(14.8)$ \\
\hline Live birth & $216(66.7)$ \\
\hline Preterm birth & $9(2.8)$ \\
\hline Take-home baby rate & $28.6 \%$ \\
\hline \multicolumn{2}{|l|}{ SD: Standard deviation } \\
\hline
\end{tabular}


lesions in the uterine cavity (17). Unsuspected intrauterine abnormalities have been diagnosed using hysteroscopy in an asymptomatic IVF population with a prevalence of as high as $50 \%$ (18). Assuming that performing hysteroscopy before IVF treatment can improve reproductive outcomes, studies of this issue have produced conflicting results and high-quality studies are lacking (19).

A meta-analysis reported by Pundir et al. (20) proved that LBR increased following hysteroscopy in women scheduled for a first IVF cycle (risk ratio: 1.30, 95\% confidence interval:1.00-1.67;

Table 2. Comparison of the patients based on different hysteroscopy and embryo transfer groups

\begin{tabular}{|c|c|c|c|c|c|}
\hline Characteristics & $\begin{array}{l}\text { Group } 1(n=488) \\
\text { (fresh, non- } \\
\text { hysteroscopy) }\end{array}$ & $\begin{array}{l}\text { Group } 2(n=101) \\
\text { (fresh, } \\
\text { hysteroscopy) }\end{array}$ & $\begin{array}{l}\text { Group } 3(\mathrm{n}=135) \\
\text { (frozen-thawed, } \\
\text { non-hysteroscopy) }\end{array}$ & $\begin{array}{l}\text { Group } 4(n=44) \\
\text { (frozen-thawed, } \\
\text { hysteroscopy) }\end{array}$ & $\mathbf{p}$ \\
\hline Age (years) & $31.4 \pm 6.2$ & $31.5 \pm 5.7$ & $30.8 \pm 5.5$ & $29.9 \pm 5.2$ & 0.365 \\
\hline Age group (years) & - & - & - & - & 0.132 \\
\hline$<20$ & $16(3.3)$ & 0 & $2(1.5)$ & $2(4.5)$ & - \\
\hline $21-25$ & $74(15.2)$ & $14(13.9)$ & $20(14.8)$ & $4(9.1)$ & - \\
\hline $26-30$ & $147(30.1)$ & $36(35.6)$ & $47(34.8)$ & $19(43.2)$ & - \\
\hline $31-35$ & $120(24.6)$ & $21(20.8)$ & $38(28.1)$ & $13(29.5)$ & - \\
\hline $36-40$ & $86(17.6)$ & $24(23.8)$ & $23(17)$ & $6(13.6)$ & - \\
\hline$>40$ & $45(9.2)$ & $6(5.9)$ & $5(3.7)$ & 0 & - \\
\hline Pregnancy result & - & - & - & - & 0.960 \\
\hline Negative & $285(58.4)$ & $58(57.4)$ & $77(57)$ & $24(54.5)$ & - \\
\hline Positive & $203(41.6)$ & $43(42.6)$ & $58(43)$ & $20(45.5)$ & - \\
\hline Pregnancy outcome & - & - & - & - & 0.402 \\
\hline No pregnancy & $285(58.4)$ & $58(57.4)$ & $77(57)$ & $24(54.5)$ & - \\
\hline Biochemical & $34(7)$ & $8(7.9)$ & $6(4.4)$ & $3(6.8)$ & - \\
\hline Abortus & $26(5.3)$ & $11(10.9)$ & $7(5.2)$ & $4(9.1)$ & - \\
\hline Live birth & $135(27.7)$ & $24(23.8)$ & $45(33.3)$ & $12(27.3)$ & - \\
\hline Preterm birth & $8(1.6)$ & 0 & 0 & $1(2.3)$ & - \\
\hline Take-home baby rate (\%) & 28.1 & 24.8 & 33.3 & 29.5 & 0.513 \\
\hline
\end{tabular}

Table 3. Comparison of the patients based on different age groups

\begin{tabular}{|c|c|c|c|c|c|c|c|}
\hline Characteristics & $\begin{array}{l}<20 \text { years } \\
(n=20)\end{array}$ & $\begin{array}{l}21-25 \text { years } \\
(n=112)\end{array}$ & $\begin{array}{l}\text { 26-30 years } \\
(n=249)\end{array}$ & $\begin{array}{l}\text { 31-35 years } \\
(n=192)\end{array}$ & $\begin{array}{l}\text { 36-40 years } \\
(n=139)\end{array}$ & $\begin{array}{l}>40 \text { years } \\
(n=56)\end{array}$ & $\mathbf{p}$ \\
\hline Hysteroscopy & - & - & - & - & - & - & 0.256 \\
\hline Not performed & $18(90)$ & $94(83.9)$ & $194(77.9)$ & $158(82.3)$ & $109(78.4)$ & $50(89.3)$ & - \\
\hline Performed & $2(10)$ & $18(16.1)$ & $55(22.1)$ & $34(17.7)$ & $30(21.6)$ & $6(10.7)$ & - \\
\hline Type of embryo transfer & - & - & - & - & - & - & 0.080 \\
\hline Fresh & $16(80)$ & $88(78.6)$ & $183(73.5)$ & $141(73.4)$ & $110(79.1)$ & $51(91.1)$ & - \\
\hline Frozen-thawed & $4(20)$ & $24(21.4)$ & 66 (26.5) & $51(26.6)$ & $29(20.9)$ & $5(8.9)$ & - \\
\hline Pregnancy result & - & - & - & - & - & - & 0.001 \\
\hline Negative & $13(65)$ & $56(50)$ & $136(54.6)$ & $103(53.6)$ & $90(64.7)$ & $46(82.1)$ & - \\
\hline Positive & $7(35)$ & $56(50)$ & $113(45.4)$ & $89(46.4)$ & $49(35.3)$ & $10(17.9)$ & - \\
\hline Pregnancy outcome & - & - & - & - & - & - & 0.001 \\
\hline No pregnancy & $13(65)$ & $56(50)$ & $136(54.6)$ & $103(53.6)$ & $90(64.7)$ & $46(82.1)$ & - \\
\hline Biochemical & $1(5)$ & $2(1.8)$ & $20(8)$ & $13(6.8)$ & $11(7.9)$ & $4(7.1)$ & - \\
\hline Abortus & $1(5)$ & $6(5.4)$ & $21(8.4)$ & $10(5.2)$ & $10(7.2)$ & 0 & - \\
\hline Live birth & $4(20)$ & $47(42)$ & $68(27.3)$ & $63(32.8)$ & $28(20.1)$ & $6(10.7)$ & - \\
\hline Preterm birth & $1(5)$ & $1(0.9)$ & $4(1.6)$ & $3(1.6)$ & 0 & 0 & - \\
\hline Take-home baby rate (\%) & 20 & 42 & 28.5 & 33.9 & 20.1 & 8.9 & $<0.001$ \\
\hline
\end{tabular}


$p=0.05$ ). The results of this meta-analysis are controversial because most studies included in this meta-analysis were non-randomized studies (20). However, another randomized controlled trial investigating LBR in a similar study population showed enhanced pregnancy rates of up to $70 \%$ following hysteroscopy (21).

By contrast with the findings of these studies, Smit et al. (13) suggested that routine hysteroscopy prior to IVF or ICSI treatments have no effect on fertility outcomes in infertile women with normal uterine cavity on transvaginal ultrasound at their multicenter randomized controlled trial. In this study, a limitation of this study was that hysteroscopy was performed by different gynecologists in several clinics because it is known that diagnostic accuracy of hysteroscopy may change depending on the operator (22). Moreover, the TROPHY trial, another randomized controlled trial evaluating the effect of hysteroscopy on LBR in women having more than two failed IVF cycles showed that hysteroscopy had no impact on LBR (10).

The reasons for conflicting results of the studies about the utility of hysteroscopy before IVF or ICSI cycles include methodological weakness and lack of quality. A recently published meta-analysis from the Cochrane database confirms this opinion. Kamath et al. (23) investigated the feasibility of routine hysteroscopy in sub-fertile women undergoing evaluation for infertility and in sub-fertile women scheduled for intrauterine insemination or IVF in this meta-analysis. After reviewing 11 publications, they concluded that there was no publication having strong evidence to support hysteroscopy as a screening method in sub-fertile women with a normal basic fertility work-up for increasing live birth and clinical pregnancy rates (23).

The important issue that has been suggested about the use and benefit of hysteroscopy before IVF is that endometrial scratching was reported to improve reproductive success rates. Although there are contradictory studies demonstrating that hysteroscopy is useful or not in this regard, a recently published randomized controlled trial and a systematic review showed that endometrial scratching does not increase pregnancy rates, and therefore larger studies with high levels of evidence are needed before they can be used in daily practice $(24,25)$.

In this study, we retrospectively assessed the pregnancy outcomes of IVF cycles applied either by fresh or FET transfers that were performed with or without hysteroscopy to evaluate the uterine cavity prior to IVF. It was demonstrated that diagnostic hysteroscopy did not improve pregnancy rate in women who underwent fresh or FET embryo transfer. There were some limitations and strengths to our study. One of the most important strengths of our study was that all the hysteroscopies were performed by a single, experienced surgeon. Hence the evaluation of the uterine cavity was consistent and should reduce once source of variability in this study. The retrospective design of the study is the limitation of our study.

\section{Conclusion}

This study has shown that performing diagnostic hysteroscopy before fresh or FET does not improve the pregnancy rates in this cohort. However, randomized-controlled prospective trials are necessary to further understand the feasibility of performing hysteroscopy before IVF or ICSI cycles.

Acknowledgement: The authors thank the participants of this study.

Ethics Committee Approval: For this study, ethical approval was taken from the Ethical Committee of Acibadem Mehmet Ali Aydinlar University (ATADEK).

Informed Consent: Written informed consent was obtained from all patients who participated in this study.

Peer-review: Externally and internally peer-reviewed.

Author Contributions: Surgical and Medical Practices: F.E., A.C.., F.B., E.B.; Concept - F.E., A.Ç.; Design - F.E., A.Ç.; Data Collection or Processing - F.E., A.Ç., A.N.A.; Analysis or Interpretation - H.G.Ç.; Literature Search - H.G.Ç., E.Ç.; Writing - F.E., H.G.Ç., E.B.

Conflict of Interest: No conflict of interest is declared by the authors.

Financial Disclosure: The authors declared that this study received no financial support

\section{References}

1. Ferraretti AP, Goossens V, de Mouzon J, Bhattacharya S, Castilla JA, Korsak V, et al; European IVF-monitoring (EIM); Consortium for European Society of Human Reproduction and Embryology (ESHRE). Assisted reproductive technology in Europe, 2008: results generated from European registers by ESHRE. Hum Reprod 2012; 27: 2571-84.

2. Simón C, Martín JC, Pellicer A. Paracrine regulators of implantation. Baillieres Best Pract Res Clin Obstet Gynaecol 2000; 14: 815-26.

3. Singh M, Chaudhry P, Asselin E. Bridging endometrial receptivity and implantation: network of hormones, cytokines, and growth factors. J Endocrinol 2011; 210: 5-14.

4. Cumming DC, Taylor PJ. Combined laparoscopy and hysteroscopy in the investigation of the ovulatory infertile female. Fertil Steril 1980; 33: 475-8.

5. Linderman H, Mohr J. CO2 hysteroscopy, diagnosis and treatment. Am J Obstet Gynecol 1976; 124: 129-33. 
6. Seshadri S, El-Toukhy T, Douiri A, Jayaprakasan K, Khalaf Y. Diagnostic accuracy of saline infusion sonography in the evaluation of uterine cavity abnormalities prior to assisted reproductive techniques: a systematic review and meta-analyses. Hum Reprod Update 2015; 21: 262-74.

7. Golan A, Eilat E, Ron-EL R Herman A, Soffer Y, Bukovsky I. Hysteroscopy is superior to hysterosalpingography in infertility investigation. Acta Obstet Gynecol Scand 1996; 75: 654-6.

8. Cunha-Filho JSL, de Souza CAB, Salazar CC, Facin AC, Freitas FM, Passos EP. Accuracy of hysterosalpingography and hysteroscopy for diagnosis of intrauterine lesions in infertile patients in an assisted fertilization programme. Gynaecol Endosc 2001; 10: 45-8.

9. Elter K, Yildizhan B, Suntay T, Kavak ZN. Diagnostic Hysteroscopy Before IVF: Which Women are Candidates? J Turk Ger Gynecol Assoc 2005; 6: 217-19.

10. El-Toukhy T, Campo R, Khalaf Y, Tabanelli C, Gianaroli L, Gordts SS, et al. Hysteroscopy in recurrent in-vitro fertilisation failure (TROPHY): a multicentre, randomised controlled trial. Lancet 2016; 387: 2614-21.

11. El-Toukhy T, Sunkara SK, Coomarasamy A, Grace J, Khalaf Y. Outpatient hysteroscopy and subsequent IVF cycle outcome: a systematic review and meta-analysis. Reprod Biomed Online 2008; 16: 712-9.

12. Di Spiezio Sardo A, Di Carlo C, Minozzi S, Spinelli M, Pistotti V, Alviggi $\mathrm{C}$, et al. Efficacy of hysteroscopy in improving reproductive outcomes of infertile couples: a systematic review and metaanalysis. Hum Reprod Update 2016; 22: 479-96.

13. Smit JG, Kasius JC, Eijkemans MJC, Koks CAM, van Golde R, Nap AW, et al. Hysteroscopy before in-vitro fertilisation (inSIGHT): a multicentre, randomised controlled trial. Lancet 2016; 387: 2622-9.

14. Pabuçcu EG, Yalçın I, Bodur T, Çağlar GS, Pabuçcu R. Impact of office hysteroscopy in repeated implantation failure: Experience of a single center. J Turk Ger Gynecol Assoc 2016; 17: 197-200.

15. Potdar N, Gelbaya T, Nardo LG. Endometrial injury to overcome recurrent embryo implantation failure: a systematic review and meta-analysis. Reprod Biomed Online 2012; 25: 561-71.

16. Yeung TW, Chai J, Li RH, Lee VC, Ho PC, Ng EH. The effect of endometrial injury on ongoing pregnancy rate in unselected subfertile women undergoing in vitro fertilization: a randomized controlled trial. Hum Reprod 2014; 29: 2474-81.

17. Demirol A, Gurgan T. Effect of treatment of intrauterine pathologies with office hysteroscopy in patients with recurrent IVF failure. Reprod Biomed Online 2004; 8: 590-4.

18. Fatemi HM, Kasius JC, Timmermans A, van Disseldorp J, Fauser BC, Devroey P, et al. Prevalence of unsuspected uterine cavity abnormalities diagnosed by office hysteroscopy prior to in vitro fertilization. Hum Reprod 2010; 25: 1959-65.

19. Rama Raju GA, Shashi Kumari G, Krishna KM, Prakash GJ, Madan K. ssessment of uterine cavity by hysteroscopy in assisted reproduction programme and its influence on pregnancy outcome. Arch Gynecol Obstet 2006; 274: 160-4.

20. Pundir J, Pundir V, Omanwa K, Khalaf Y, El-Toukhy T. Hysteroscopy prior to the first IVF cycle: a systematic review and meta-analysis. Reprod Biomed Online 2014; 28: 151-61.

21. Elsetohy KA, Askalany AH, Hassan M, Dawood Z. Routine office hysteroscopy prior to ICSI vs. ICSI alone in patients with normal transvaginal ultrasound: a randomized controlled trial. Arch Gynecol Obstet 2015; 291: 193-9.

22. Kasius JC, Broekmans FJ, Veersema S, Eijkemans MJ, van Santbrink EJ, Devroey P, et al. Observer agreement in the evaluation of the uterine cavity by hysteroscopy prior to in vitro fertilization. Hum Reprod 2011; 26: 801-7.

23. Kamath MS, Bosteels J, D'Hooghe TM, Seshadri S, Weyers S, Mol BWJ, et al. Screening hysteroscopy in subfertile women and women undergoing assisted reproduction. Cochrane Database Syst Rev 2019; 4: CD012856.

24. van Hoogenhuijze NE, Kasius JC, Broekmans FJM, Bosteels J, Torrance HL. Endometrial scratching prior to IVF; does it help and for whom? A systematic review and meta-analysis. Hum Reprod Open 2019; 2019: hoy025.

25. Lensen S, Osavlyuk D, Armstrong S, Stadelmann C, Hennes A, Napier E, et al. A Randomized Trial of Endometrial Scratching before In Vitro Fertilization. N Engl J Med 2019; 380: 325-34. 